\title{
NCCN Roundtable Tackles Disparities in Cancer Care
}

\section{Abstract}

During a roundtable on disparities in cancer care at the recent NCCN 22nd Annual Conference: Improving the Quality, Effectiveness, and Efficiency of Cancer Care, panelists from a variety of disciplines brought professional and personal experiences to the discussion of eliminating cancer disparities and reducing differences in incidence and mortality rates among minority populations. The panel agreed that these issues must be addressed through research in prevention, screening, clinical trials, and equal access to quality care.

J Natl Compr Canc Netw 2017;15(5.5):671-675

The NCCN 22nd Annual Conference: Improving the Quality, Effectiveness, and Efficiency of Cancer Care convened during a week when Congress was expected to vote on the American Health Care Act (AHCA), the Republican-sponsored replacement plan for the Patient Protection and Affordable Care Act (ACA). Ultimately, however, House Republicans pulled the AHCA from a vote, leaving the future of the ACA unclear. The timing of this rendered even more relevance to the topic of an NCCN Roundtable: "Emerging Issues in Oncology-Addressing Health Disparities in Cancer Care From Diagnosis to Survivorship." Panelists from a variety of disciplines brought professional and personal experiences to the discussion. All agreed that despite recent advances in cancer care, health disparities still exist and could even deepen.

Moderator Clifford Goodman, PhD, Senior Vice President of The Lewin Group, kicked off the roundtable by expressing concerns. "I'm worried about healthcare disparities in America," he said. "I'm worried that we are perhaps on the cusp of things, if not getting worse, adding risk to the system. Today, decisions could be made on [Capitol] Hill that could put already disadvantaged populations at greater risk. It's important to have a nationally recognized panel of experts...to address these issues... These messages we are hearing today come with great credibility."

\section{What Does Disparity Look Like?}

Shauntice Allen, PhD, Assistant Professor of Environmental Health Sciences, University of Alabama School of Public Health, pointed out that issues of disparity reflect an "interplay between economics, cultural, and social factors.” Anne Filipic, President, Enroll America, a nonprofit organization that promotes health coverage, said that disparities are a natural consequence of a lack in health coverage.

Edith Mitchell, MD, Associate Director, Diversity Programs, and Director, Center to Eliminate Cancer Disparities, Sidney Kimmel Medical College at Thomas Jefferson University, added that genomics figures into the problem, because some subgroups also have higher rates of certain cancers, and being disadvantaged only makes treatment less likely.

Phyllis Pettit Nassi, MSW, Manager of Special Populations, Huntsman Cancer Institute at the University of Utah, highlighted disparities based on geography, noting that Native American tribes and others living in "rural or frontier" areas often lack access to care and, therefore, are inadequately treated. Moon S. Chen Jr, PhD, MPH, Associate Director, Cancer Control, University of California Davis Comprehensive Cancer Center, added that certain subgroups based on age, race, and socioeconomic status are poorly represented in clinical trials.

In short, the panel described how disparities exist not only among subsets of Americans typically thought to be short-changed, but also among other individuals based on factors such as age, insurance coverage, and even zip code. These gaps in care should be fully acknowledged, Dr. Allen urged. "We need to be able to have honest conversations about differences in class, culture, and social experiences," she said. "Until we are able to, I think we'll continue to see disparities." 


\section{Meet the Panelists}

Clifford Goodman, PhD, moderator: Senior Vice President at the Lewin Group, a premier national healthcare and human services consulting firm based in Falls Church, Virginia.

Shauntice Allen, PhD: Assistant Professor in the Department of Environmental Health Sciences in the University of Alabama School of Public Health. Dr. Allen has vast experience with health education program design, implementation, and evaluation, and she is currently living with metastatic breast cancer.

Moon S. Chen Jr, PhD, MPH: Associate Director for Cancer Control at the University of California Davis Comprehensive Cancer Center. Dr. Chen is a nationally renowned expert in cancer health disparities, leading a portfolio of research that addresses determinants of cancer risk and their mitigation in human populations.

Anne Filipic: President of Enroll America, a nonprofit organization dedicated to maximizing the number of Americans who enroll in and retain health coverage through the Affordable Care Act. She was formerly Deputy Director at the White House Office of Public Engagement and Deputy Director of Intergovernmental Affairs for the former Health and Human Services Secretary, Kathleen Sebelius.

Edith Mitchell, MD: Clinical Professor, Department of Medicine and Medical Oncology, and Program Leader of Gastrointestinal Oncology at Sidney Kimmel Medical College at Thomas Jefferson University. She is also Associate Director for Diversity Programs and Director of the Center to Eliminate Cancer Disparities.

Phyllis Pettit Nassi, MSW: Manager of Special Populations at Huntsman Cancer Institute at the University of Utah. A member of the Cherokee Nation, Ms. Pettit Nassi has studied cultural and social implications on underserved populations and has worked with their representatives, organizations, and academic institutions for more than 30 years.

\section{Coverage Impacts Disparity}

The ACA proposed to close gaps in health disparities and health coverage. "Today, 7 years after its passage, we have seen $>22$ million Americans gain health coverage," said Ms. Filipic.

Across all 50 states and across all demographic groups, the uninsured rate has dropped. "We've seen tremendous gains," she noted, also pointing out that these gains are at risk "as the future of the ACA is in question." She expects that lower-income and sicker individuals "stand to lose the most" under the proposed ACA repeal efforts. Even consumers who can retain coverage may see higher deductibles, which poses grave concerns for patients with high medical needs, she said.

"It sounds as if disparities arise on social and legal events," Dr. Goodman commented, "political activities bear upon access."

Ms. Filipic discussed the issue of paying for health coverage, which borders on impossible for some individuals. She spoke of focus groups during the initial ACA enrollment period. "One thing that stuck with me is that people don't even check out their options when they believe they can't afford any of them. As one women said, 'You don't go shoe shopping if you can't afford shoes."' Dr. Allen agreed, noting, "Cost is a real barrier."

Ms. Filipic also pointed to "cultural competency," or lack of it, as a component in failure to seek coverage. "Just because a person can enroll for coverage doesn't mean they are aware of their options or will take action... This is particularly important in communities of color," she said. She has learned that for previously uninsured consumers to enroll in the ACA, the availability of in-person application assistance is critical. In communities of color, individuals are twice as likely to want personal help, she noted.

Dr. Mitchell added that financial toxicity is not just a problem for people of low socioeconomic status. It's also apparent among "individuals who earn a regular salary and have good insurance," she said, pointing out that for some care, a $20 \%$ copay is still more than many persons - whether working or not-can afford.

\section{A View From a Different System}

Ms. Pettit Nassi is not only involved in outreach to Native American populations, she is herself a member of the Cherokee Nation. In asking about this dual perspective, Dr. Goodman noted, "It's not always the same America."

The Indian Health Service is underfunded and its population is highly underserved, Ms. Pettit Nassi said. "For every dollar that's requested, they get 18 to 34 cents. It's a fiscal issue that most cancer [care] providers really don't understand. In small tribes, you wipe out the entire budget for the year with one cancer diagnosis."

In fact, the Indian Health Service is currently operating under a "life or limb" protocol, which means that aside from basic clinic services, only 
"threats to life and limb" are assured funding. In this situation, physicians may not even screen for cancer. "I am told, 'because we don't treat it, we don't look for it," she said.

This means that cancer prevention efforts can be largely futile. An individual may be persuaded to undergo a mammogram or colonoscopy, but they often cannot find a location that provides those screenings. Dr. Goodman said this indicates, "there are resourcesbased biases and people are affected by the context around them."

\section{Genomics Impacts Disparity}

Dr. Mitchell pointed out that disparities are not always obvious - they can be hiding in our genes. She noted the value of understanding how cancer genomics defines certain populations and contributes to disparities.

Both triple-negative breast cancer (an aggressive subtype) and colorectal cancer are more common among African Americans than Caucasians and occur at a younger age. Recognition of this difference can raise awareness about prevention and screening. For example, she said, colorectal cancer screening should begin at age 45 years for African Americans, but coverage for screening is often denied for individuals who fall outside of the typical age-based guidelines.

To Dr. Mitchell, genomic risk can be addressed by "taking what we already know and applying it to the right populations." Dr. Chen agreed, "We need to recognize there are already racial/ethnic groups for which cancer is the leading cause of death, and it's soon to be for all Americans. We just need to look into our mirror and recognize that health disparity is among us. We have opportunities as health professionals to address this by applying what we know."

Because lung cancer is the leading cause of cancer death, the "biggest bang for the buck" in applying current knowledge is probably the promotion of smoking cessation programs, Dr. Chen continued. Vaccination against human papillomavirus is also a worthwhile effort. Through the application of knowledge and strategies that are already established, "We can harness the power of prevention, promote abatement of cancer through screening, and ameliorate suffering by improving quality of life," he suggested.

\section{Zip Code Is a Good Identifier}

Rural and frontier communities are not the only geographic locations to suffer from health disparities. Urban zip codes also matter, said Dr. Mitchell. "In fact, if you look at risk factors for disparity, zip code is one of the best identifiers," she said.

"Know your patient population!" Dr. Mitchell advised attendees. "Adapt your healthcare system to your community."

This includes understanding issues in the community and the cultural and financial factors at play, identifying resources that can be tapped, and getting community leaders involved in promoting health care services. For example, she noted that the Sidney Kimmel Medical College at Thomas Jefferson University has extended hours for chemotherapy infusion and walk-in clinics, giving patients with time constraints more flexible access to treatment.

To "synergize with other resources in the community," she said, the healthcare system must "break our silos." Ms. Filipic, who also commented on this topic, suggested that providers should take advantage of "trusted messengers in the community" and empower them to help with outreach. "This not only helps build the village that is necessary," she explained, "but it's also a powerful message to the audience we're trying to reach."

Dr. Mitchell indicated that the AJCC has published a document that aids healthcare institutions in interacting with the community to identify needs and resources. "You don't have to do it all yourself," she told listeners. "Just get out of the tower and discover the needs and resources in the community."

Ms. Pettit Nassi agreed: "If we don't commit to making a difference in changing health disparities by leaving our silos, by going out into the community to really see for ourselves, then we will fail."

\section{Excluding Clinical Trial Participation}

The panelists all said they have seen certain subgroups of patients either directly or indirectly excluded from clinical trials, a type of bias that potentiates disparities in cancer care. This is unfortunate, because clinical trial participation is one means of assuring good care at little to no cost to the patient, Dr. Allen pointed out.

"If a provider cannot speak the patient's language, there's hesitation to spend the time needed 
to explain the benefits of a clinical trial," Dr. Chen said. "There is also perhaps a predisposition to think this person would not accept participation."

Native Americans and Alaskans, in particular, "are being completely left behind," Ms. Pettit Nassi said, and the gap is widening as cancer care moves into genomics and precision medicine.

According to Dr. Chen, failure to include a broader population translates into trial data that are less applicable in the real world. "Clinical trials are the gold standard for assessing efficacy of therapy, and they are only as good as the population that participates," he emphasized.

Dr. Goodman questioned whether some racial or ethnic groups might actually have their own reservations about enrollment. "Are there concerns among minority populations about being pulled into trials?" he asked.

Dr. Chen acknowledged that the term "clinical trial" is ambiguous and has "inherent implications" for some groups. "Does it mean an 'experiment'? Does it mean going through the 'legal system'? And how do we explain 'clinical'? The challenge is translating this term into a language the patient understands," he responded.

The panel suggested that non-English-speaking patients and those with low levels of education are not the only ones who might have reservations about clinical trial participation. Dr. Allen, who is highly informed and educated, said she would be reluctant to join another clinical trial after participating in one that was demanding. "I was surprised by the time commitment. As a busy young professional, it was a lot," she said.

She also believes that strict eligibility requirements are a problem for patients who may want to participate but do not quite fit the profile. "I understand the importance of rigor," she shared, but noted that waiting for tumors to grow, or even hoping a current drug fails because patients need to have had so many lines of therapy before they can enroll, "can be a real issue for people," she said.

\section{Does Professional Bias Play a Role?}

One cannot get away from the observation that even oncology providers contribute to disparities by exerting bias, the panelists agreed.
Dr. Allen offered a glimpse of this bias as seen from her perspective as both researcher and patient with cancer. "I have a very good relationship with my oncologist and have access to lots of information. I have seen other individuals spoken to [differently] and offered something different. It's probably not meant to inherently discourage someone, but it occurs."

"We have conscious and unconscious bias," Dr. Mitchell, a gastrointestinal oncologist, acknowledged. "We make assumptions about individuals. For example, we think that patients of a certain age will not want treatment, and that's certainly not the case for all. It's the same with some racial and ethnic populations."

Ms. Pettit Nassi discussed how both professional and resource bias can affect disparities. She told of a recent project after which a colleague noted, "When I see an Indian, I see diabetes. I need to see an Indian woman and think gynecologic cancer." However, Ms. Pettit Nassi also noted, "should they see that, they don't have the resources to treat it."

"Bias arising from a lack of healthcare resources may redirect what the physician offers," Dr. Goodman noted.

\section{Individualization Is Key}

Part of the solution is for providers to see each patient as unique, the panelists agreed. "We need to make sure we are giving individualized medicine," Dr. Mitchell said. "Make sure our care is based on the individual-not what they look like or where they live."

She relayed an anecdote about a 93-year-old female patient. The woman asked, "Dr. Mitchell, are you going to tell me about my options for treating this cancer?" to which she replied, "Yes, of course! Why do you ask that?" The patient answered, "Another doctor told me to get my papers in order, go home, and pray, and I told him, 'Young man, I came here for cancer care. I'll decide when I need to pray!"'

To Dr. Mitchell, this story speaks to the need to avoid forming biases based on age, skin color, language, and zip code. Dr. Allen added, "Remember, your patients are real people; they want to be involved in their caregiving. Listen to them. Give them the opportunity to do that." 
Disparities in Cancer Care

In wrapping up, many of the panelists urged those in attendance to make their voices heard in Congress and at the state level as lawmakers shape health policy.

"Apply what you learn about the community to what we know about prevention, screening, and treatment, and work with the community to address the needs of the community," Dr. Mitchell said. "If you have an opinion regarding what is happening in Washington today, call your congressional representative today...so your voice can be heard." 